\title{
Effect of Age on Swallowing Parameters in an Electroglottographic Study
}

\author{
${ }^{1}$ Samriddha Kumar, ${ }^{2}$ Indranil Chatterjee, ${ }^{3}$ Arpita C Shahi, ${ }^{4}$ Bhaskar Dey, ${ }^{5}$ Amitabha Roychoudhury
}

\begin{abstract}
Aim: To establish age as an essential factor in the assessment of swallowing

Materials and methods: Sixty subjects were divided into two age groups (18 to 33 years and 55 to 70 years) and were further divided into males and females. Each subject was made to swallow four times each with different food consistency (dry, water, puree, and solid). The electroglottograph (EGG) was done during the swallowing process, and each swallow was depicted by a peak and a trough in the EGG. The X-axis in the EGG showed time while the $Y$-axis shows percentage amplitude. With the help of web plot digitizer software, the change in time and the change in percentage amplitude were obtained from the point of onset and offset of the peak and trough in the graph and the highest and lowest point on the peak and trough in the graph respectively.
\end{abstract}

Results: A significant effect of age on dry swallow time and percentage amplitude, water swallow time and percentage amplitude, puree swallow time and percentage amplitude, solid swallow time and percentage amplitude was obtained.

Conclusion: The results indicated that as age increased there was a significant increase in the change in time and a significant decrease in the change in percentage amplitude parameter.

Clinical significance: Age should be considered as an important factor in the process of assessment of swallowing. The altered physiology with an increase in age should influence the clinician's perception of normalcy and prognostic predictions.

Keywords: Age, Dry swallow, Electroglottograph (EGG), Percentage amplitude, Pure swallow, Solid swallow, Swallowing, Time, Water swallow.

How to cite this article: Kumar S, Chatterjee I, Shahi AC, Dey B, Roychoudhury A. Effect of Age on Swallowing Parameters in an Electroglottographic Study. Int J Phonosurg Laryngol 2018;8(1):13-18.

Source of support: Nil

Conflict of interest: None

\section{INTRODUCTION}

Swallowing occurs as an organized physiological process that sends saliva or ingested material from the mouth to

${ }^{1}$ Postgraduate Student (MASLP), ${ }^{2}$ Lecturer, Speech and Hearing, ${ }^{3,4}$ Ex. Clinical Supervisor, ${ }^{5}$ Guest Lecturer

${ }^{1-5}$ Ali Yavar Jung National Institute of Speech and Hearing Disabilities Diviyangjan RC, Kolkata, West Bengal, India

Corresponding Author: Samriddha Kumar, Postgraduate Student (MASLP), Ali Yavar Jung National Institute of Speech and Hearing Disabilities Diviyangjan RC, Kolkata, West Bengal, India, e-mail: samriddhakumar211@gmail.com the stomach. This process occurs typically so smoothly and effortlessly that it belies the complexity of the neuromuscular coordination that is responsible for the swallowing sequence. Generally, swallowing rate elicited by saliva is carried on at a rate of about $0.5 \mathrm{~mL} /$ minute and must be either swallowed or expectorated. The elevated basal rate of swallowing while the person is alert leads to about 1000 swallows per day. ${ }^{1}$

\section{Effect of Age on Swallowing Physiology}

Although there are very few studies yet some of the researchers have come up with significant findings. ${ }^{2}$ Describes hyolaryngeal excursion chiefly accounted for the longer durations for swallowing with increased age and bolus consistency.

Perlman et al. found that the duration of oropharyngeal pressure was affected by age, gender, and bolus consistency that is adult subjects to have higher pressures than young subjects. ${ }^{3}$

Hiss et al. found elderly adults had longer seasonal affective disorder (SAD) than young and middle-aged adults concerning saliva swallows; males showed a reduction in SAD with increasing age while females exhibited an increase in SAD with increasing age. ${ }^{4}$

The above findings contrasted with the findings of Susan et al. ${ }^{5}$

Steele et al. stated that significantly more variability in lingual kinematics patterns was found in the older subject group. ${ }^{6}$

\section{Electroglottographic Swallowing Studies}

Sorin et al. describe the feasibility of using the EGG to evaluate aspects of the swallow was assessed in six nonimpaired, two Parkinsonism, and two dysphagic subjects. Low-frequency tissue impedance difference across the neck could be recorded with an EGG during swallowing. On six normal subjects, EGG waveforms were consistently monophasic and appeared to be in the same time with laryngeal height changes. The waveforms of the two dysphagic subjects were different considerably from those of the controls, and their timing and shapes were agreeable with videofluoroscopic observations. ${ }^{7}$ 
Perlman and Grayhack, explained EGG as a noninvasive, electrical impedance device that was developed for finding vocal fold contact during phonation, could also be used to detect maximum laryngeal displacement and the length of laryngeal movement during swallowing. ${ }^{8}$

Perlman and Liang, studied the frequency response of a modified fourcin EGG at frequencies for the slow varying laryngeal movement of swallowing and compared those findings with the response characteristics of the EGG at frequencies for phonation. ${ }^{9}$

Schultz, used EGG to examine temporal relationships of laryngeal movement, oropharyngeal pressure and submental muscle contraction during swallowing.

Nozaki et al., also explained the importance of EGG in an assessment of swallowing. ${ }^{10,11}$

Ding showed how surface electromyography (EMG) had been used effectively in teaching patients to swallow maneuvers in clinical intervention settings. ${ }^{12}$

Farooq, presented an approach that uses an EGG instrument for an objective and automatic detection of food intake. He concluded EGG to be better than acoustic assessment methods for swallowing. ${ }^{13}$

\section{AIMS AND OBJECTIVES}

The study aims to evaluate the effect of age on nonpathological swallowing physiology of different food consistencies.

\section{MATERIALS AND METHODS}

This study is done on descriptive research design. The study determines the electroglottographic recordings during swallow in 2 age ranges (18 to 33 years mean age 22.07 years, standard deviation 2.9 and 55 to 70 years mean age 59.63 years, standard deviation 4.7), in 4 oral intake consistencies (dry, water, puree and solid).

Both the age groups consisted of equal number of males and females. A written consent was taken from the subjects informing them about the detailed methodology, outcome, and purpose of the research and permission for further use of the data for analysis and publication.

\section{Inclusion Criteria}

Healthy volunteers with the adequately normal oral transition time absence of any abnormality on oral and glottal anatomy and physiology including voice problems confirmed by perceptual assessment.

\section{Exclusion criteria}

Chronic or acute voice disorders, prolonged or permanent tracheostomy and/or respiratory infections gastroesophageal reflux disease (GERD) and any pulmonary disease [chronic obstructive pulmonary disease (COPD), Acute respiratory distress syndrome (ARDS) and/or Asthma)], history of drug or substance abuse, psychiatric problem, cerebrovascular accidents, head injury, any obesity or overweight symptoms, pregnancy or breastfeeding period, dermatological problem or metal allergy, repaired cleft palate and/or lip or submucous cleft palate.

The four consistencies: Dry swallow-swallowing one's saliva (own saliva is used), water-swallowing commercially available mineral water (commercially available drinking water is used), puree-swallowing foods that are prepared to a smooth consistency by grinding and then pureeing them. Appearance is smooth like pudding (4 table spoons of commercially available Horlicks mixed in half cup of water is used), solid-swallowing bite-size foods that are cut into pieces no larger than $0.5^{\prime \prime} \times 0.5^{\prime \prime}$ 0.5 "(commercially available hard biscuits are used).

\section{Instrumentation}

The electroglottography, Tiger DRS, Inc. was used for electroglottography with calibration as per the company manual. The two electrodes with the surface area of 8.04 $\mathrm{cm}^{2}$ and with a $4 \mathrm{~cm}$ distance between them are placed on the two thyroid lamina allowing the subject to speak and breathe naturally comfortably. The instrument is set on the EGG mode and in highest EGG gain, and lowest microphone gain. EG-PC3 EGG system with the software Dr Speech software, Tiger DRS Inc. Vocal Assessment for Windows, Version-4.30; 1998. Tiger DRS, Inc. has been developed by Daniel Zaoming Huang, Cindy Chen, David Yuan, Robert $\mathrm{O}^{\prime}$ Brien; program developed by Steven Z, and DSP by TOM $Q$ was used for the study. The Webplotdigitozer Software Version 3.12 Released (June 3, 2017) is free software available in the internet, copyright by Ankit Rohatgi, was used to obtain values on different points on the graph.

\section{Procedure}

Case history and oromotor examination followed by laryngoscopy to comply within the inclusion and exclusion criteria. Detailed oromotor examination of anatomy and physiology of lips, tongue, hard palate, soft palate, mandible, teeth, and uvula were checked. A swallowing trial with $5 \mathrm{ml}$ of water was done to measure oral transition time.

The subjects were instructed, "I will tie this band with electrodes on your throat you will swallow within one second from the cue I will provide you. You will have to swallow three times each for each food item given to you including the initial dry swallow. You are expected to feel the gulp go down your throat." 
Once the three trials for each swallow is taken, the time window of 1 second is selected, and the amplitude percentage ranged from -100 to +100 microvolts. The graph is changed into a JPG format by taking a print screen and cropping the rest of the visual display leaving out the graph and saving this cropped image in Microsoft Paint. The prominent peak representing the change in impedance during the swallow is identified. The glottal closure during the swallow is assumed to reduce the impedance across the two electrodes in the two laminas. The image is fed into the Webplotdigitizer software. The distance between the points on the graphical representation was used to obtain the values.

Descriptive statistics to find the mean and standard deviation values of change in time and change in percentage amplitude difference for swallowing of the four food consistencies for the two age groups and the two gender groups. One way analysis of variance (ANOVA) was done to see if there is a significant difference between the two age groups. One way ANOVA was done to see if there is a significant difference between the two gender groups. One way repeated measures ANOVA was done to see if there is a significant difference between the four food consistencies.

\section{RESULTS}

Values of change in time and change in percentage amplitude during swallowing for the two gender and two age groups for the four consistencies:

\section{DISCUSSION}

A vivid literature search has depicted a dearth of literature in the field of electroglottographic swallowing studies except for the studies by Sorin and Farooq. There is a novelty in the procedure of using different consistency foods as well as concerning compari ng the results of varying age groups in using electroglottography in the assessment of swallowing. Age has previously been mentioned many times to be crucial in the process of the evaluation of swallowing yet in clinical practice we often miss out considering this point. The results from the study have been found to be comparable and agreeable will previous studies on swallowing using conventional assessment procedures FEES and VFS. The detailed discussions of the results of my study have been provided herewith.

Demographic data of the change in glottal impedance during swallowing of four food consistencies in two age groups containing individuals of both genders.

The demographic data of the change in glottal impedance was studied under two parameters of change in time and change in percentage amplitude.

\section{Change in Time}

Graph 1 shows that irrespective of age, the change in time increases as we move from dry to solid consistency with the increase in viscosity. It also indicates that an increase in age increases the change in time irrespective of the food consistency. The findings from this study as shown in Table 1 have been found to agree with the conclusions of the study by Robbins, 1992 where he stated that oropharyngeal swallow took considerably longer time in the oldest age group than in any other group because of the delayed initiation of hyolaryngeal excursion for the swallow of different textures in both genders. The findings in the present study are also in agreement with the investigation by Hiss, where he has found greater swallowing apnea duration in older group than the younger group and in females more than in males in all the age groups when four different volumes of bolus have been used. ${ }^{14}$

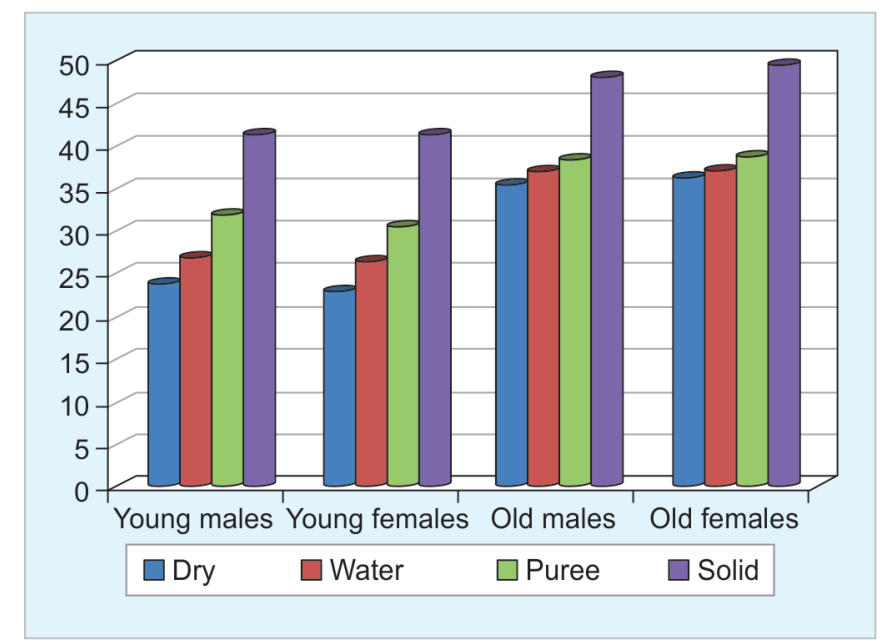

Graph 1 : Change in time for all subjects considering the differences in age for both genders in four food consistencies (each unit on Y-axis represents 2 milliseconds)

Table 1: Change in time of swallow in the two gender and two age groups for the four consistencies (each unit being equal to 2 milliseconds)

\begin{tabular}{lllllllll}
\hline & \multicolumn{3}{c}{$\begin{array}{c}\text { Young female } \\
\text { Mean and } \pm \text { S.D }\end{array}$} & Young male (units) & $\begin{array}{l}\text { (units) } \\
\text { (units) }\end{array}$ & \multicolumn{2}{c}{$\begin{array}{l}\text { Old female } \\
\text { (units) }\end{array}$} \\
\hline Dry & 23.78 & 3.08 & 22.97 & 3.04 & 35.45 & 3.20 & 36.63 & 2.97 \\
Water & 26.89 & 1.79 & 26.47 & 2.71 & 36.93 & 2.59 & 37.18 & 3.13 \\
Puree & 31.95 & 3.32 & 30.49 & 2.71 & 38.54 & 2.14 & 38.78 & 3.33 \\
Solid & 41.46 & 2.97 & 41.64 & 2.28 & 48.20 & 3.10 & 49.62 & 2.14 \\
\hline
\end{tabular}




\section{Change in Percentage Amplitude}

Graph 2 shows that irrespective of age, the change percentage amplitude decreases from dry to puree with an increase in viscosity. An exception is seen only in dry swallow in old females. It also indicates that an increase in age decreases the change in percentage amplitude irrespective of the food consistency. Hiss in his study showed that while females exhibited an increase in swallowing apnea duration with an increase in age, males show a decrease in swallowing apnea duration with an increase in age. While other studies do not support this, the finding could indicate a rather gradual glottal closure taking more time. The findings as shown in Table 2 are consistent with the study by Nilsson et al. in 1996 which infers that age-related changes in nerves and muscles hamper muscle strength and coordination of swallowing and may lead to poorer glottal closure as an effect of age

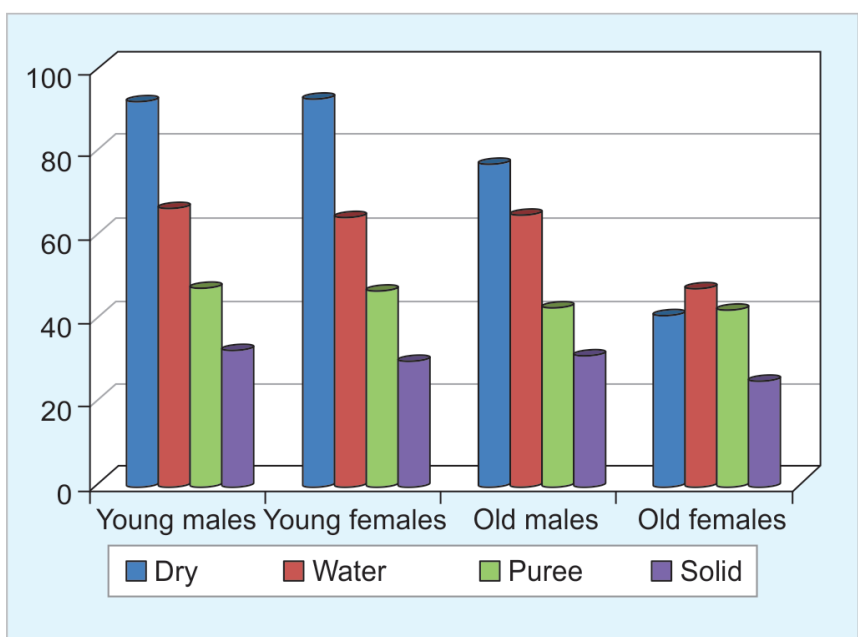

Graph 2: Change in percentage amplitude for all subjects considering the differences in age for both genders in four food consistencies (each unit on the $\mathrm{Y}$-axis represents $2 / 3$ microvolts) thus explaining the reduction in percentage amplitude for old males as found in the present study. ${ }^{15}$

\section{Effect of Age on Glottal Impedance Change during Swallowing}

The effect of age on change in glottal impedance was studied under two parameters of change in time and change in percentage amplitude.

\section{With Respect to Change in Time}

Graph 3 shows that as the age increases the change in time increases. All the four consistencies show the same pattern. The difference in change in time is more in the dry swallow and progressively increases as we move towards solid food consistency. This finding as shown in Table 3 is consistent with the conclusions of the study by Treole in 2001 where the authors have compared between six age

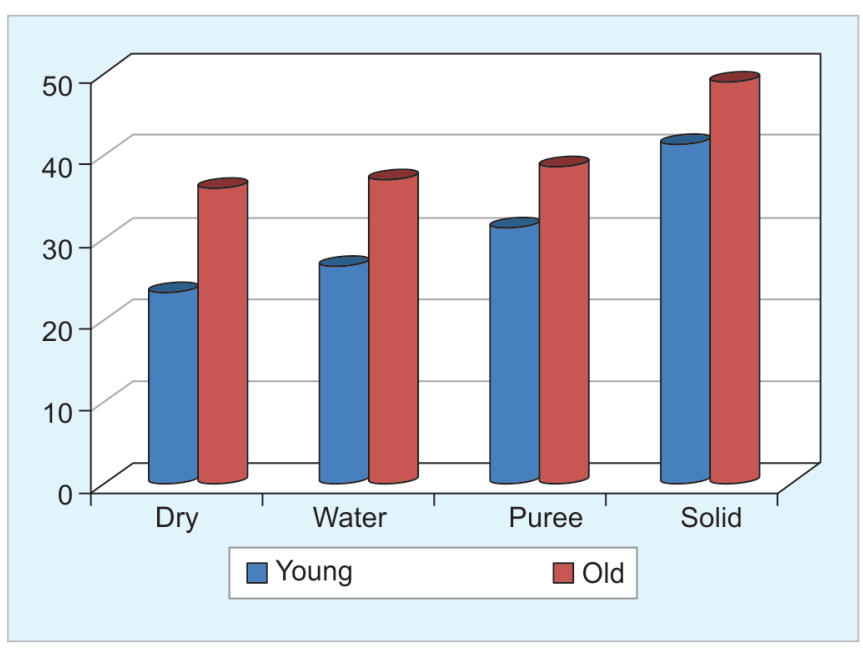

Graph 3: Change in time for the two age groups (each unit on Y-axis represents two milliseconds)

Table 2: Change in percentage amplitude of swallow in the two gender and two age groups for the four consistencies (each unit being equal to $2 / 3$ microvolts)

\begin{tabular}{|c|c|c|c|c|c|c|c|c|}
\hline \multirow{2}{*}{$\begin{array}{l}\text { Mean and } \pm \text { S.D. } \\
\text { Dry }\end{array}$} & \multicolumn{2}{|c|}{$\begin{array}{l}\text { Young male } \\
\text { (units) }\end{array}$} & \multicolumn{2}{|c|}{$\begin{array}{l}\text { Young female } \\
\text { (units) }\end{array}$} & \multicolumn{2}{|c|}{$\begin{array}{l}\text { Old male } \\
\text { (units) }\end{array}$} & \multicolumn{2}{|c|}{$\begin{array}{l}\text { Old female } \\
\text { (units) }\end{array}$} \\
\hline & 91.66 & 6.16 & 92.81 & 5.70 & 77.36 & 6.14 & 40.40 & 4.41 \\
\hline Water & 65.63 & 5,97 & 64.37 & 5.69 & 64.54 & 7.95 & 46.18 & 4.72 \\
\hline Puree & 46.49 & 5.27 & 45.81 & 8.88 & 41.88 & 6.17 & 41.74 & 8.32 \\
\hline Solid & 31.74 & 6.15 & 29.03 & 6.06 & 30.36 & 3.38 & 24.29 & 6.31 \\
\hline
\end{tabular}

Table 3: Effect of age on different swallows

\begin{tabular}{llllllllll}
\hline & & \multicolumn{3}{l}{ Sum of squares } & df & \multicolumn{2}{l}{ Mean square } \\
\cline { 3 - 9 } & & $B G$ & WG & BG & WG & BG & WG & $F$ & Sig. \\
\hline \multirow{2}{*}{ Dry } & Time & 2405.147 & 455.013 & 1 & 58 & 2405.147 & 9.380 & 256.425 & 0.000 \\
& amplitude & 16689.007 & 12039.344 & 1 & 58 & 16689.007 & 207.575 & 80.400 & 0.000 \\
\multirow{3}{*}{ Pater } & Time & 1615.751 & 380.584 & 1 & 58 & 1615.751 & 6.562 & 246.236 & 0.000 \\
& amplitude & 1393.848 & 4690.585 & 1 & 58 & 1393.848 & 80.872 & 17.235 & 0.000 \\
\multirow{3}{*}{ Solid } & Time & 830.750 & 493.276 & 1 & 58 & 830.750 & 8.505 & 97.681 & 0.000 \\
& amplitude & 282.491 & 2996.701 & 1 & 58 & 282.491 & 51.667 & 5.467 & 0.023 \\
& Time & 811.220 & 415.175 & 1 & 58 & 811.220 & 7.158 & 113.327 & 0.000 \\
& amplitude & 140.148 & 2093.780 & 1 & 58 & 140.148 & 36.100 & 3.882 & 0.054 \\
\hline
\end{tabular}


ranges and four intake volumes. The study states that the swallowing apnea duration was found to be more in the elderly population than in young group irrespective of the intake volumes. The study by Robbins, stated that oropharyngeal swallow took longer time considerably in the oldest age group than in any other group because of the delayed initiation of hyolaryngeal excursion for the swallow of different textures in both genders. A p-value of $<0.05$ was obtained when comparing the transition times of the two age groups. Sonies et al. in his study also states that for the elderly of age more than 55 years have increased oropharyngeal pressure duration associated with the increased effort to glottal adduction. This can be a reason for such an increased change in time for the elderly as observed in this study. ${ }^{16}$ Selley et al., 1989 , in his study showed an increase in swallow apnea duration seen in 33 elderly subjects than young controls went the respiratory pattern across swallowing of liquids is assessed. ${ }^{17}$

\section{With Respect to Change in Percentage Amplitude}

Graph 4 shows that as age increases the change in percentage amplitude decreases. All the four consistencies show the same pattern. The difference in the change in percentage amplitude is most significant in the dry swallow as compared to the other three consistencies. It should also be noted that in most of the elderly subjects multiple swallows were observed after the initial intake although instructions of a single swallow was given. The above findings may be attributed to the week glottal adduction and hyolaryngeal excursion due to age related changes in muscles and nerves. The findings are consistent with the study by Nilsson et al. in 1996 which infers that age-related changes in nerves and muscles hamper muscle strength and coordination of swallowing. As they assessed 53 elderly individuals they found

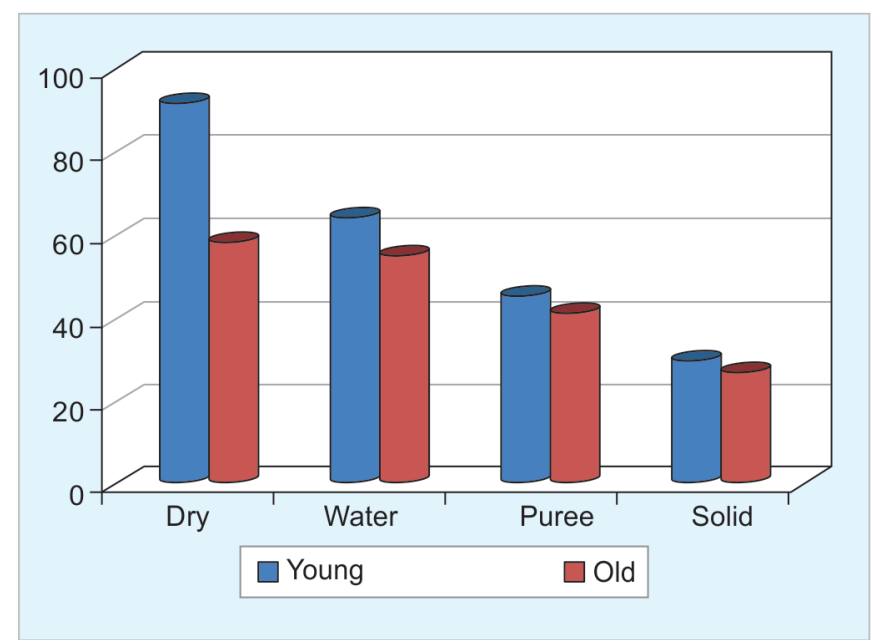

Graph 4: Change in percentage amplitude for the two age groups (each unit on $\mathrm{Y}$-axis represents $2 / 3$ microvolts) significant differences between the young and elderly group. The study found the following significant features of elderly swallow: decreased peak suction pressure, increased frequency of multiple swallows after one ingestion, increased frequency of polyphasic laryngeal movements, increased frequency of inspiration after swallowing, and increased frequency of coughing during or after swallowing. Sheth et al. in her study describe the changes in non-pathological elderly subjects when compared to a young group. She finds that there is a decrease in the strength and there is an altered sequence of pharyngeal contractions. ${ }^{18}$

\section{CONCLUSION}

- There was a significant increase in change in time with the increase in age.

- There was a significant decrease in the change in percentage amplitude with the increase in age.

\section{CLINICAL SIGNIFICANCE}

Age should be considered as an important factor in the process of assessment of swallowing. The altered physiology with the increase in age should influence the clinician's perception of normalcy and prognostic predictions.

\section{REFERENCES}

1. Dodds WJ, Stewart ET, Logemann JA. Physiology and radiology of the normal oral and pharyngeal phases of swallowing. AJR. American journal of roentgenology. 1990 May;154(5):953963.

2. Robbins J, Hamilton JW, Lof GL, Kempster GB. Oropharyngeal swallowing in normal adults of different ages. Gastroenterology. 1992 Sep 1;103(3):823-829.

3. Schultz JL, Perlman AL, VanDaele DJ. Laryngeal movement, oropharyngeal pressure, and submental muscle contraction during swallowing. Archives of physical medicine and rehabilitation. 1994 Feb 1;75(2):183-188.

4. Hiss SG, Strauss M, Treole K, Stuart A, Boutilier S. Effects of age, gender, bolus volume, bolus viscosity, and gustation on swallowing apnea onset relative to lingual bolus propulsion onset in normal adults. Journal of Speech, Language, and Hearing Research. 2004 Jun 1;47(3):572-583.

5. Donzelli J, Brady S, Wesling M, Craney M. Simultaneous modified Evans blue dye procedure and video nasal endoscopic evaluation of the swallow. The Laryngoscope. 2001 Oct;111(10):1746-1750.

6. Steele CM, Van Lieshout PH. Influence of bolus consistency on lingual behaviors in sequential swallowing. Dysphagia. 2004 Aug 1;19(3):192-206.

7. Sorin R, McClean MD, Ezerzer F, Meissner-Fishbein B. Electroglottographic evaluation of the swallow. Archives of physical medicine and rehabilitation. 1987 Apr;68(4): 232-5.

8. Perlman AL, Grayhack JP. Use of the electroglottograph for measurement of temporal aspects of the swallow: preliminary observations. Dysphagia. 1991 Jun 1;6(2):88-93. 
9. Perlman AL, Liang H. Frequency response of the Fourcin electroglottograph and measurement of temporal aspects of laryngeal movement during swallowing. Journal of Speech, Language, and Hearing Research. 1991 Aug 1;34(4):791795.

10. Nozaki S, Kang J, Miyai I, Matsumura T. Electroglottographic evaluation of swallowing in Parkinson's disease. Rinsho shinkeigaku= Clinical neurology. 1994 Sep;34(9):922924.

11. Nozaki S, Matsumura T, Takahashi M, Miyai I, Kang J. Electroglottographic studies in myasthenia gravis patients- -swallowing in exacerbation and remission stage. Rinsho shinkeigaku= Clinical neurology. 1997 Sep;37(9):841-844.

12. Ding R, Larson CR, Logemann JA, Rademaker AW. Surface electromyographic and electroglottographic studies in normal subjects under two swallow conditions: normal and during the Mendelsohn manuever. Dysphagia. 2002 Jan 1;17(1): $1-2$.
13. Farooq M, Fontana JM, Sazonov E. A novel approach for food intake detection using electroglottography. Physiological measurement. 2014 Mar 26;35(5):739.

14. Hiss SG, Treole K, Stuart A. Effects of age, gender, bolus volume, and trial on swallowing apnea duration and swallow / respiratory phase relationships of normal adults. Dysphagia. 2001 Mar 1;16(2):128-135.

15. Nilsson H, Ekberg O, Olsson R, Hindfelt B. Quantitative aspects of swallowing in an elderly nondysphagic population. Dysphagia. 1996 Jun 1;11(3):180-184.

16. Sonies BC, Parent LJ, Morrish K, Baum BJ. Durational aspects of the oral-pharyngeal phase of swallow in normal adults. Dysphagia. 1988 Mar 1;3(1):1-10.

17. Selley WG, Flack FC, Ellis RE, Brooks WA. Respiratory patterns associated with swallowing: Part 1. The normal adult pattern and changes with age. Age and ageing. 1989 May 1;18(3):168-172.

18. Sheth N, Diner WC. Swallowing problems in the elderly. Dysphagia. 1988 Dec 1;2(4):209-215. 